\title{
Diffusion-Based Height Analysis Reveals Robust Microswimmer-Wall Separation
}

\author{
Stefania Ketzetzi, ${ }^{1}$ Joost de Graaf $\odot{ }^{2},{ }^{2}$ and Daniela J. Kraft $\odot^{1}$ \\ ${ }^{1}$ Soft Matter Physics, Huygens-Kamerlingh Onnes Laboratory, Leiden University, P.O. Box 9504, 2300 RA Leiden, Netherlands \\ ${ }^{2}$ Institute for Theoretical Physics, Center for Extreme Matter and Emergent Phenomena, Utrecht University, \\ Princetonplein 5, 3584 CC Utrecht, Netherlands
}

(Received 11 June 2020; accepted 8 October 2020; published 2 December 2020)

\begin{abstract}
Microswimmers typically move near walls, which can strongly influence their motion. However, direct experimental measurements of swimmer-wall separation remain elusive to date. Here, we determine this separation for model catalytic microswimmers from the height dependence of the passive component of their mean-squared displacement. We find that swimmers exhibit "ypsotaxis," a tendency to assume a fixed height above the wall for a range of salt concentrations, swimmer surface charges, and swimmer sizes. Our findings indicate that ypsotaxis is activity induced, posing restrictions on future modeling of their stilldebated propulsion mechanism.
\end{abstract}

DOI: 10.1103/PhysRevLett.125.238001

Confining surfaces, such as planar walls, have a farreaching impact in the microswimmer world, often ensuring microswimmer function and survival [1]. Encounters with surfaces give rise to accumulation, as seen for sperm [2], algae [3], and bacteria [4], and enable the formation of bacterial biofilms that facilitate their spreading, cooperation, and capture of nutrients [5-7]. Moreover, surfaces can significantly modify swimming trajectories; e.g., bacteria often exhibit circular motion with the direction controlled by the boundary condition [8-11], in stark contrast to their run-and-tumble motion in bulk.

Striking surface effects are not only found in biological systems, but are also present for synthetic microswimmers [12-18]. Model catalytic colloidal swimmers exhibit autonomous directed motion due to self-generated chemical gradients [19]. Recently, neighboring walls were shown to significantly alter the magnitude of their swim speeds [15-18]. This revealed that walls play a far greater than previously expected role on self-propulsion, providing a path toward resolving seemingly conflicting experimental observations. For example, speed differences under similar conditions may stem from the phoretic interplay between the hydrodynamic boundary condition on the wall and the out-of-equilibrium chemical species generated by the swimmer [17]. Current models predict a wide range of behaviors close to walls, including hovering, sliding, and forward and/or backward propulsion [12,20-33]. This diversity is partly due to the complexity of and uncertainties

Published by the American Physical Society under the terms of the Creative Commons Attribution 4.0 International license. Further distribution of this work must maintain attribution to the author(s) and the published article's title, journal citation, and DOI. in the propulsion mechanism and partly due to the hydrodynamic and numerous phoretic couplings that wall proximity can introduce. Thus, quantitative insight into swimmer-wall separation is pivotal to pinpointing missing details of the propulsion mechanism and, in turn, tailoring swimming behaviors, e.g., for guiding microswimmers in complex environments.

To date, no reported experiment has directly measured swimmer-wall separations. However, based on qualitative observations, separations are anticipated to be smaller than the swimmer size [13,34], even as small as a few tens of nanometers [35,36]. Such separations cannot be directly resolved by standard optical microscopy [35], which is why holographic microscopy has been proposed [16], as it yields three-dimensional positions of spherical particles with high precision [37]. However, fitting holograms of spheres half-coated with a metal is computationally expensive, especially when studying dynamics, since discrete dipole approximations have to be employed in the numerical calculations to obtain their positions [38]. Furthermore, inhomogeneities in the metal coating introduce additional fit parameters and uncertainties in determining particle positions. Another way to measure small particle-wall separations is total internal reflection microscopy, which yields separations from the scattering of evanescent waves off of particles close to a wall [39]. Here, too, the asymmetric coating interferes with interpreting the result and obtaining accurate measurements. Hence, a novel measuring approach is needed.

In this Letter, we present a facile and straightforward method for obtaining microswimmer-wall separations in situ. We determine the translational diffusion coefficient of the swimmer from mean-squared displacement curves and obtain the height from its theoretically predicted dependence on swimmer-wall separation. Our method 

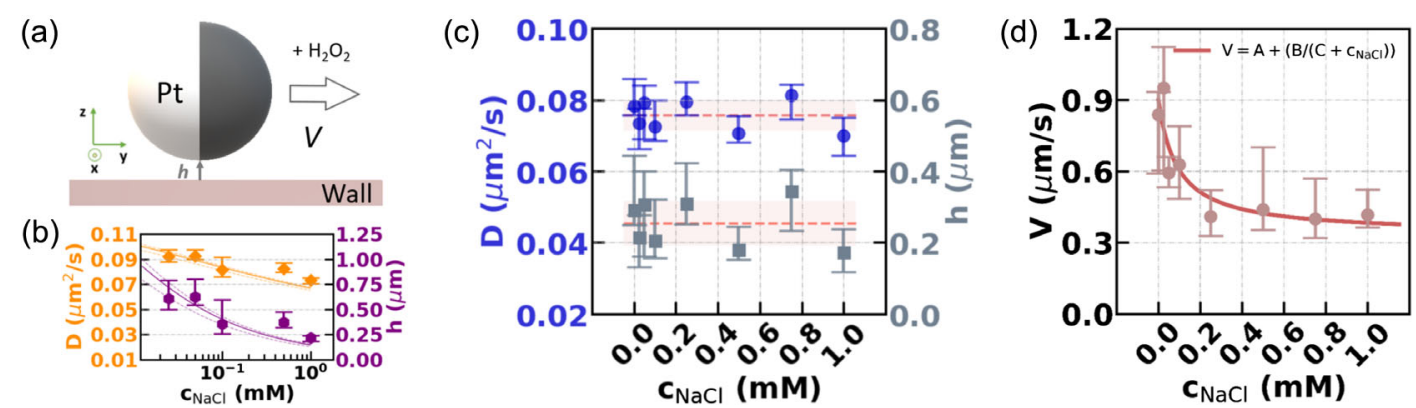

FIG. 1. Salt-dependent motion above a planar wall. (a) Schematic of the experiment. We obtain the swimmer-wall separation $h$ from the measured translational diffusion coefficient $D$ of the swimmer and its theoretically predicted dependence on wall separation. (b)-(d) Effect of salt concentration $c_{\mathrm{NaCl}}$ on the motion of $2.77 \pm 0.08 \mu \mathrm{m}$ colloids with $4.4 \pm 0.2 \mathrm{~nm}$ Pt. All reported values are medians, and error bars denote first quartiles. (b) Diffusion coefficient (orange diamonds) and separation (purple hexagons) in the Brownian state in water with $c_{\mathrm{NaCl}}$. Lines show theoretical predictions based on balancing electrostatics and gravity. (c) Diffusion coefficient (circles) and separation (squares) in the active state in aqueous $10 \% \mathrm{H}_{2} \mathrm{O}_{2}$ with $c_{\mathrm{NaCl}}$. Dotted lines indicate mean values. (d) Speed decrease in $10 \%$ $\mathrm{H}_{2} \mathrm{O}_{2}$ with $c_{\mathrm{NaCl}}$. The solid line is a least-squares fit with $V=A+\left[B /\left(C+c_{\mathrm{NaCl}}\right)\right]$, where $A$ is $0.35 \pm 0.09 \mu \mathrm{m} / \mathrm{s}$, the remaining speed in high salt, $B$ is a prefactor, and $C$ is $0.09 \pm 0.07 \mathrm{mM}$, the ion concentration already present in solution, following from ionic diffusioosmosis along the wall.

can be applied to most synthetic microswimmers and may be extended to a range of swimming microorganisms, moving parallel to walls. We applied it here to catalytically propelled model microswimmers. Besides the fuel concentration, we systematically varied additional parameters known to affect self-propulsion, as well as particle-wall separations in passive systems: the salt concentration in solution, swimmer size, and swimmer zeta potential. We were thereby able to gain unprecedented insights into their effect and the presence of a wall on the swimming behavior.

We obtained swimmer-wall separations from experimental measurements of the separation-dependent translational diffusion coefficient $D$ of the swimmers. $D$ as well as propulsion speeds $V$ were extracted from mean squared displacements (MSDs) following Refs. [40,41]. That is, we fitted the short-time regime $\left(\Delta t \ll \tau_{R}\right)$ of the MSDs with $\Delta r^{2}=4 D \Delta t+V^{2} \Delta t^{2}$ [40]; $\tau_{R}$ is the rotational diffusion time, $\tau_{R}=1 / D_{R, \text { bulk }}$, with $D_{R \text {,bulk }}=k_{B} T / 8 \pi \eta R^{3}$ the bulk rotational diffusion coefficient, $R$ the radius, $\eta$ the viscosity, $k_{B}$ the Boltzmann constant, and $T$ the absolute temperature. The first term corresponds to the passive diffusion contribution that is usually obscured by the activity-induced, short-time ballistic behavior [41] but may be obtained with sufficient statistics. Reliable measurements require framerate adjustment, such that the regime where both diffusion and activity contribute to the MSD can be resolved. See Supplemental Material [42], which additionally includes Refs. [43-47], for details on tracking and MSD calculation [48], as well as a discussion on the consistent short-time $\left(\Delta t \ll \tau_{R}\right)$ expansion of the MSD [40,41,49]. To calculate the separation $h$ between the particle and wall [see also Fig. 1(a)], we first consider the ratio $d=D / D_{\text {bulk }}$, with $D_{\text {bulk }}=k_{B} T / 6 \pi \eta R$ the bulk diffusion constant. For $d \gtrsim 0.6$, the well-known prediction by Faxén [50-53]: $d(h)=1-(9 / 16) \gamma+(1 / 8) \gamma^{3}-(45 / 256) \gamma^{4}-(1 / 16) \gamma^{5}$, with $\gamma=R /(h+R)$, can be used to extract $h$. For $d \lesssim 0.4$, a lubrication theory result, $d(h)=-1 /[(8 / 15) \log (h / R)-$ $0.9588]$, is more appropriate $[54,55]$. In the intermediate $(0.4 \lesssim d \lesssim 0.6)$ regime, applicable to most of our experiments, we interpolate the combined numerical data by O'Neill [55] and Kezirian [56]; see Supplemental Material, Sec. II A [42]. The $d(h)$ relation that follows is provided as a supplement to this publication. Here, we fitted for $h$ using the interpolated expression.

In all experiments, we used 3-(trimethoxysilyl)propyl methacrylate (TPM) monodisperse colloids [57] half-coated with a thin Pt layer $(\approx 4.5 \pm 0.2 \mathrm{~nm})$ at a dilute concentration $\left(\approx 10^{-7} \mathrm{vol} / \mathrm{vol}\right)$. In water, colloids exhibited passive Brownian motion, while dispersion in $10 \% \mathrm{H}_{2} \mathrm{O}_{2}$ rendered them active through a catalytic process. Colloids quickly reached the lower glass wall and continued to move adjacent to it, while their motion was recorded with an inverted Nikon Eclipse Ti microscope through a $60 \times$ oil objective $(\mathrm{NA}=1.4)$. Swimming experiments were recorded for 30 s at 19 frames per second; see Supplemental Material, Sec. I C [42].

To demonstrate the effectiveness of our method, we first carried out control experiments in deionized water and in

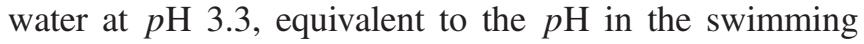
experiments, at 5 frames per second. In these cases, $D$ was acquired from fitting MSDs with $\Delta r^{2}=4 D \Delta t$. Figure 1(b) shows that the extracted separation corresponds well to a theoretical prediction based on a balance of electrostatic repulsion and gravity [58,59]; see Supplemental Material, Sec. II B [42]. That is, we recovered the expected decrease in separation with increasing salt concentration: Salt increases the solution's ionic strength, thereby effectively screening the charge on the particle and wall. This reduces the Debye length, i.e., the distance over which surface charges act, bringing the colloids closer to the wall. 

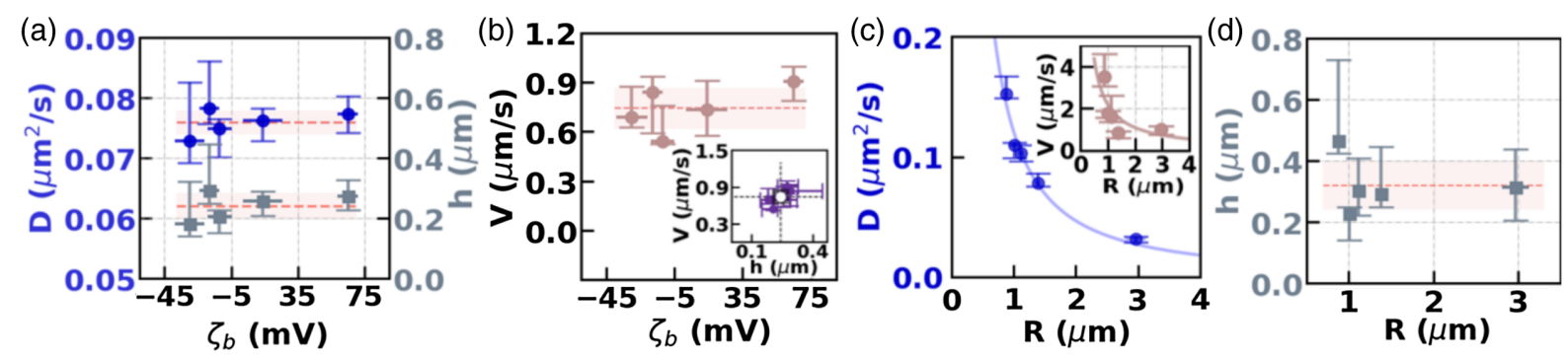

FIG. 2. Swimmer base zeta potential and size dependence of propulsion above a planar wall. All reported values are medians, error bars denote first quartiles, and dotted lines represent mean values. All experiments were performed in aqueous $10 \% \mathrm{H}_{2} \mathrm{O}_{2}$. (a), $(\mathrm{b}) \mathrm{The}$ base zeta potential $\zeta_{b}$ of TPM colloids with diameters between $2.70 \pm 0.06$ and $2.77 \pm 0.08 \mu \mathrm{m}$ and Pt coating thicknesses $\approx 4.4 \pm$ $0.2 \mathrm{~nm}$ was varied through surface functionalization. (a) Diffusion coefficient (circles) and separation (squares) with $\zeta_{b}$. (b) Speed for the same $\zeta_{b}$ range as in (a). The inset shows speed with separation (purple circles), with the white circle marking the intersection of mean values and the rectangle around it denoting standard deviations. (c),(d) Variation of the radius $R$ of active TPM spheres with similar $\zeta_{b}$ and $\mathrm{Pt}$ (coating thickness $\approx 4.5 \pm 0.2 \mathrm{~nm}$ ) affects (c) the diffusion coefficient and (d) swimmer-wall separation. The inset in (c) shows swim speed with $R$. Solid lines in (c) are fits with $a / R^{b}$ with $a=0.120 \pm 0.004 \mu \mathrm{m}^{3} / \mathrm{s}$ and $b=1.3 \pm 0.2$ (main) and the expected $a / R$ [29] with $a=2.2 \pm 0.4 \mu \mathrm{m}^{2} / \mathrm{s}$ (inset). The dotted line in (d) shows mean separation $(0.32 \pm 0.08 \mu \mathrm{m})$.

To verify our method further, we compared separations resulting from our diffusion coefficient-based method to those directly measured with digital in-line holographic microscopy, for uncoated silica spheres with well-known size and refractive index [60]. We found good agreement between the two methods, which confirmed that we indeed recovered colloid-wall separations, using a computed rather than a measured value of $D_{\text {bulk }}$.

Having established the validity of our method, we employed it to our catalytic microswimmers. First, we studied the effect of salt concentration in solution. For these experiments, we used TPM spheres of $2.77 \pm 0.08 \mu \mathrm{m}$ diameter half-coated with $4.4 \pm 0.2 \mathrm{~nm}$ Pt. Surprisingly, in active systems we found a behavior completely unlike that of passive systems in Fig. 1(b). For the same particles and salt concentration range, $D$ and $h$ remain constant within measurement precision; see Fig. 1(c). Particles propel themselves parallel to the wall at constant separations of $0.25 \pm 0.06 \mu \mathrm{m}$.

At the same time, we found a decrease in speed with increasing salt concentration [see Fig. 1(d)], where the line represents the least-squares fit with $V=A+$ $\left[B /\left(C+c_{\mathrm{NaCl}}\right)\right]$. This expression follows from a saltgradient-based contribution to the observed speed [34], with $A$ the remaining speed in the limit of high salt, $B$ a prefactor, and $C$ the ion concentration already present in the medium. From the fit, we find the reasonable numbers $0.35 \pm 0.09 \mu \mathrm{m} / \mathrm{s}$ and $0.09 \pm 0.07 \mathrm{mM}$, for $A$ and $C$, respectively. The fitted $C$ value agrees reasonably well with the background ion concentration $(0.008 \mathrm{mM})$ we obtained from electrical conductivity measurements [61] for $10 \%$ $\mathrm{H}_{2} \mathrm{O}_{2}(2.7 \mu \mathrm{S} / \mathrm{cm}$, Ilium technology, model 2100 conductivity meter) assuming hydrogen ions as the dominant ion species. We return to this salt gradient in the discussion.

Second, we explored the effect of colloid zeta potential $\zeta$, the electric potential at the colloid's surface. We used $2.70 \pm 0.06$ and $2.77 \pm 0.08 \mu \mathrm{m}$ diameter colloids with different surface functionalizations [62] and, thus, different $\zeta$. The reported $\zeta$ correspond to those of the parent colloids (see Supplemental Material, Secs. I A and IB [42], for characterization) before adding the Pt coating. We therefore use the adjective "base" and a subscript "b," i.e., $\zeta_{b}$, to indicate that we know only the zeta potential of the uncoated colloid and not that of the swimmer. We note that passive colloids with $\zeta_{b}>-12 \mathrm{mV}$ were typically stuck on the negatively charged wall; see also Supplemental Material, Sec. ID [42].

However, for the active system, we found that wall separation remained unaffected for the entire (wide) range of $\zeta_{b}$ under study; see Fig. 2(a). In all cases, particles moved at $0.24 \pm 0.04 \mu \mathrm{m}$ from the wall, which matches the separations measured for different salt concentrations. Unexpectedly, as we will return to, the colloids self-propelled not only at a constant $h$ when varying $\zeta_{b}$, but also at quantitatively comparable speeds $V$; see Fig. 2(b). We can indeed collapse the data by plotting $V$ as a function of $h$ [see the inset in Fig. 2(b)], further demonstrating that $\zeta_{b}$ does not affect the swimming behavior. We note that the direction of motion was away from the Pt cap for both positive and negative $\zeta_{b}$.

Third, we focused on swimmer size, another parameter known to affect swim speeds [29]. We performed experiments using TPM spheres with a wide range of radii but with similar Pt coating thicknesses and $\zeta_{b}$; see Supplemental Material, Sec. I A, for characterization [42]. We found that diffusion coefficient decreases with swimmer size; see Fig. 2(c), where the solid line represents the least-squares fit with $D=$ $a / R^{b} \quad\left(a=0.120 \pm 0.004 \mu \mathrm{m}^{3} / \mathrm{s} \quad\right.$ and $\left.\quad b=1.3 \pm 0.2\right)$. The inset shows the measured swim speeds together with a fit of the expected scaling $V=a / R$ [29] ( $a=2.2 \pm$ $0.4 \mu \mathrm{m}^{2} / \mathrm{s}$ ). Strikingly, swimmer-wall separation remained relatively constant with $R$; see Fig. 2(d) - the dashed line shows the mean separation of $0.32 \pm 0.08 \mu \mathrm{m}$. 

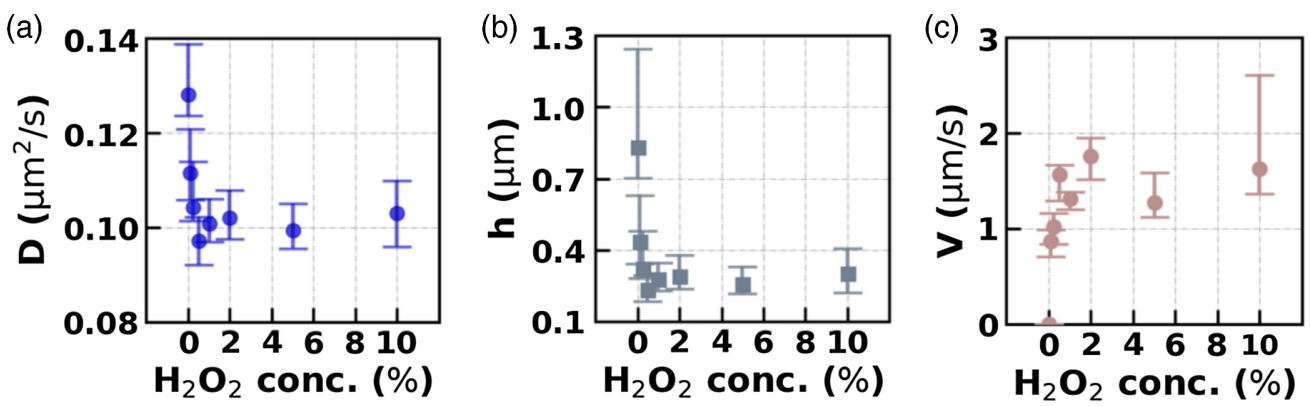

FIG. 3. Activity-induced ypsotaxis. (a) Diffusion coefficient $D$ with increasing $\mathrm{H}_{2} \mathrm{O}_{2}$ (fuel) concentration. (b) Equivalent swimmerwall separation $h$ for the same fuel concentration range. (c) Swim speed $V$ for the same fuel concentration range as in (a),(b). All experiments were performed using $2.23 \pm 0.11 \mu \mathrm{m}$ diameter TPM spheres with $4.5 \pm 0.2 \mathrm{~nm}$ Pt. All reported values are medians and error bars denote first quartiles.

The above experiments reveal that our swimmers exhibit "ypsotaxis": a tendency to assume a specific height (in Greek: ypsos) for a wide range of parameters. Remarkably, the height appears independent of salt concentration, $\zeta_{b}$, and even size, running not only counter to our intuition for passive systems but also to features of common selfpropulsion mechanisms. For our Pt-coated swimmers, this robust separation distance was found to be $0.27 \pm 0.11 \mu \mathrm{m}$ on average, in line with the observation that micron-sized catalytic swimmers do not self-propel over steps of a few hundred nanometers [13]. Such a height is further consistent with wall-dependent speeds [15-18], for which wall separation must not substantially exceed the swimmer size to ensure strong osmotic coupling $[12,20,26]$. The wide range of swimmer sizes employed here showed that buoyancy is not the prime contributor to ypsotaxis. This is further underpinned by our observation of swimmers moving along the top wall, upon inversion of the sample holders, for a period of time. We hypothesize that ypsotaxis is instead primarily caused by phoretic and osmotic flows; i.e., it is activity driven.

To test for this, we performed experiments using $2.23 \pm$ $0.11 \mu \mathrm{m}$ diameter TPM spheres with $4.5 \pm 0.2 \mathrm{~nm}$ Pt for various $\mathrm{H}_{2} \mathrm{O}_{2}$ concentrations and, hence, degrees of activity. Indeed, we found that the diffusion coefficient and, thus, swimmer-wall separation not only decrease rapidly with increasing fuel concentration from the Brownian state $(0 \%$ $\mathrm{H}_{2} \mathrm{O}_{2}$ ) [see Figs. 3(a) and 3(b), respectively], but also plateau beyond $0.25 \% \mathrm{H}_{2} \mathrm{O}_{2}$. Similarly, the speed also increases sharply and then plateaus above $0.25 \% \mathrm{H}_{2} \mathrm{O}_{2}$; see Fig. 3(c). These observations imply that the constant separation distance is induced by the activity, thereby confirming our hypothesis on the origin of ypsotaxis. We argue that said origin also causes the active alignment of catalytic swimmers with respect to the wall $[12,13,42,63]$; see the discussion in Supplemental Material, Sec. II D [42].

Our results provide new insights into the debated nature of the propulsion mechanisms [64]. Current thinking favors self-electrophoresis [34,65], i.e., motion generated via selfgenerated ionic currents, as simple salts are known to greatly decrease propulsion speeds. The lack of speed variation with $\zeta_{b}$, however, is not commensurate with this or other ion-based propulsion mechanisms, typically scaling with $\zeta$ or $\zeta^{2}$; see [66]. A possible explanation is that a different $\zeta$ at the Pt cap dominates the swimmer's behavior.

However, speed variation with salt-typically indicative of a change in activity - is not readily reconciled with a constant $h$ which is also activity driven, even if the cap's $\zeta$ dominates. Drawing upon our previous work [17], we provide an alternative wall-centric explanation: Suppose that the swimmer's bulk speed is unaffected by adding salt. The swimmer's effective near-wall speed may still vary, provided salt impacts the osmotic counterflow induced by the swimmer-generated chemical species interacting with the wall [17]. Our fit in Fig. 1(d) reveals that the osmotic contribution to the speed bears the hallmarks of ionic diffusion [67]. This requires a net-neutral gradient of ions with different electric mobilities to be involved, often referred to as a salt gradient. This salt gradient might originate from the chemical dissociation reactions in the long-range $\mathrm{H}_{2} \mathrm{O}_{2}$ gradient with the wall [66], stemming from fuel consumption at the Pt cap. This model would have the right features to show an ionic diffusio-osmosis along the wall; see Supplemental Material, Sec. II C [42].

In summary, we established a novel method for measuring microswimmer-wall separations utilizing the height dependence of the diffusive component of their meansquared displacement. We found that catalytic model microswimmers propel at roughly fixed heights of a few hundred nanometers from planar walls. Our work further showed that nearby walls could be dominant factors in controlling swim speeds; i.e., ion-induced flow may play a role only at the wall and not at the swimmer surface. This would necessitate a paradigm shift in modeling experimental observations and in identifying the still missing details of their propulsion mechanism. Our method can be readily applied to other types of spherical microswimmers moving parallel to walls and may be extended to different swimmer shapes as well. We are confident that further application of our method will provide novel insights on the 
impact of confining surfaces in the microswimmer world and, in turn, facilitate predicting swimming behaviors in complex environments.

We gratefully acknowledge Rachel Doherty for providing TPM colloids and for discussions on colloid functionalizations. We thank Ruben Verweij and Nikos Oikonomeas for useful discussions on holographic microscopy and Aidan Brown for discussions on the propulsion mechanism and for pointing out a relevant passage in the literature. J. d. G. thanks NWO for funding through StartUp Grant No. 740.018.013 and through association with the EU-FET project NANOPHLOW (766972) within Horizon 2020. D. J. K. gratefully acknowledges funding from the European Research Council (ERC) under the European Union's Horizon 2020 research and innovation program (Grant Agreement No. 758383).

[1] J. Elgeti, R. G. Winkler, and G. Gompper, Physics of microswimmers - single particle motion and collective behavior: A review, Rep. Prog. Phys. 78, 056601 (2015).

[2] L. Rothschild, Non-random distribution of bull spermatozoa in a drop of sperm suspension, Nature (London) 198, 1221 (1963).

[3] V. Kantsler, J. Dunkel, M. Polin, and R. E. Goldstein, Ciliary contact interactions dominate surface scattering of swimming eukaryotes, Proc. Natl. Acad. Sci. U.S.A. 110, 1187 (2013).

[4] A. P. Berke, L. Turner, H. C. Berg, and E. Lauga, Hydrodynamic Attraction of Swimming Microorganisms by Surfaces, Phys. Rev. Lett. 101, 038102 (2008).

[5] H.-C. Flemming, J. Wingender, U. Szewzyk, P. Steinberg, S. A. Rice, and S. Kjelleberg, Biofilms: An emergent form of bacterial life, Nat. Rev. Microbiol. 14, 563 (2016).

[6] C. Berne, C. K. Ellison, A. Ducret, and Y. V. Brun, Bacterial adhesion at the single-cell level, Nat. Rev. Microbiol. 16, 616 (2016).

[7] H. Flemming and S. Wuertz, Bacteria and archaea on earth and their abundance in biofilms, Nat. Rev. Microbiol. 17, 247 (2019).

[8] W. R. DiLuzio, L. Turner, M. Mayer, P. Garstecki, D. B. Weibel, H. C. Berg, and G. M. Whitesides, Escherichia coli swim on the right-hand side, Nature (London) 435, 1271 (2005).

[9] E. Lauga, W. R. DiLuzio, G. M. Whitesides, and H. A. Stone, Swimming in circles: Motion of bacteria near solid boundaries, Biophys. J. 90, 400 (2006).

[10] L. Lemelle, J.-F. Palierne, E. Chatre, and C. Place, Counterclockwise circular motion of bacteria swimming at the air-liquid interface, J. Bacteriol. 192, 6307 (2010).

[11] D. Lopez and E. Lauga, Dynamics of swimming bacteria at complex interfaces, Phys. Fluids 26, 071902 (2014).

[12] S. Das, A. Garg, A. I. Campbell, J. Howse, A. Sen, D. Velegol, R. Golestanian, and S. J. Ebbens, Boundaries can steer active janus spheres, Nat. Commun. 6, 8999 (2015).

[13] J. Simmchen, J. Katuri, W. E. Uspal, M. N. Popescu, M. Tasinkevych, and S. Sánchez, Topographical pathways guide chemical microswimmers, Nat. Commun. 7, 10598 (2016).

[14] A. T. Brown, I. D. Vladescu, A. Dawson, T. Vissers, J. Schwarz-Linek, J.S. Lintuvuori, and W. C. K. Poon, Swimming in a crystal, Soft Matter 12, 131 (2016).

[15] M. Wei, C. Zhou, J. Tang, and W. Wang, Catalytic micromotors moving near polyelectrolyte-modified substrates: The roles of surface charges, morphology, and released ions, ACS Appl. Mater. Interfaces 10, 2249 (2018).

[16] A. L. Holterhoff, M. Li, and J. G. Gibbs, Self-phoretic microswimmers propel at speeds dependent upon an adjacent surface's physicochemical properties, J. Phys. Chem. Lett. 9, 5023 (2018).

[17] S. Ketzetzi, J. de Graaf, R. P. Doherty, and D. J. Kraft, Slip Length Dependent Propulsion Speed of Catalytic Colloidal Swimmers near Walls, Phys. Rev. Lett. 124, 048002 (2020).

[18] M. Heidari, A. Bregulla, S. M. Landin, F. Cichos, and R. von Klitzing, Self-propulsion of janus particles near a brushfunctionalized substrate, Langmuir 36, 7775 (2020).

[19] S. Ebbens, R. A. L. Jones, A. J. Ryan, R. Golestanian, and J. R. Howse, Self-assembled autonomous runners and tumblers, Phys. Rev. E 82, 015304(R) (2010).

[20] W. E. Uspal, M. N. Popescu, S. Dietrich, and M. Tasinkevych, Guiding Catalytically Active Particles with Chemically Patterned Surfaces, Phys. Rev. Lett. 117, 048002 (2016).

[21] M. N. Popescu, S. Dietrich, and G. Oshanin, Confinement effects on diffusiophoretic self-propellers, J. Chem. Phys. 130, 194702 (2009).

[22] D. G. Crowdy, Wall effects on self-diffusiophoretic janus particles: A theoretical study, J. Fluid Mech. 735, 473 (2013).

[23] T.-Y. Chiang and D. Velegol, Localized electroosmosis (leo) induced by spherical colloidal motors, Langmuir 30, 2600 (2014).

[24] W. E. Uspal, M. N. Popescu, S. Dietrich, and M. Tasinkevych, Self-propulsion of a catalytically active particle near a planar wall: From reflection to sliding and hovering, Soft Matter 11, 434 (2015).

[25] W.E. Uspal, M. N. Popescu, S. Dietrich, and M. Tasinkevych, Rheotaxis of spherical active particles near a planar wall, Soft Matter 11, 6613 (2015).

[26] Y. Ibrahim and T. B. Liverpool, The dynamics of a selfphoretic janus swimmer near a wall, Eur. Phys. Lett. 111, 48008 (2015).

[27] A. Mozaffari, N. Sharifi-Mood, J. Koplik, and C. Maldarelli, Self-diffusiophoretic colloidal propulsion near a solid boundary, Phys. Fluids 28, 053107 (2016).

[28] Z. Shen, A. Würger, and J. S. Lintuvuori, Hydrodynamic interaction of a self-propelling particle with a wall comparison between an active janus particle and a squirmer model, Eur. Phys. J. E 41, 39 (2018).

[29] S. Ebbens, M.-H. Tu, J. R. Howse, and R. Golestanian, Size dependence of the propulsion velocity for catalytic janus-sphere swimmers, Phys. Rev. E 85, 020401(R) (2012).

[30] M. N. Popescu, W. E. Uspal, A. Domínguez, and S. Dietrich, Effective interactions between chemically 
active colloids and interfaces, Acc. Chem. Res. 51, 2991 (2018).

[31] S. E. Spagnolie and E. Lauga, Hydrodynamics of selfpropulsion near a boundary: Predictions and accuracy of far-field approximations, J. Fluid Mech. 700, 105 (2012).

[32] J. S. Lintuvuori, A. T. Brown, K. Stratford, and D. Marenduzzo, Hydrodynamic oscillations and variable swimming speed in squirmers close to repulsive walls, Soft Matter 12, 7959 (2016).

[33] M. Kuron, P. Stärk, C. Holm, and J. de Graaf, Hydrodynamic mobility reversal of squirmers near flat and curved surfaces, Soft Matter 15, 5908 (2019).

[34] A. Brown and W. Poon, Ionic effects in self-propelled Pt-coated janus swimmers, Soft Matter 10, 4016 (2014).

[35] D. Takagi, A. B. Braunschweig, J. Zhang, and M. J. Shelley, Dispersion of Self-Propelled Rods Undergoing FluctuationDriven Flips, Phys. Rev. Lett. 110, 038301 (2013).

[36] D. Takagi, J. Palacci, A. B. Braunschweig, M. J. Shelley, and J. Zhang, Hydrodynamic capture of microswimmers into sphere-bound orbits, Soft Matter 10, 1784 (2014).

[37] S.-H. Lee, Y. Roichman, G.-R. Yi, S.-H. Kim, S.-M. Yang, A. van Blaaderen, P. van Oostrum, and D. G. Grier, Characterizing and tracking single colloidal particles with video holographic microscopy, Opt. Express 15, 18275 (2007).

[38] A. Wang, T. G. Dimiduk, J. Fung, S. Razavi, I. Kretzschmar, K. Chaudhary, and V. N. Manoharan, Using the discrete dipole approximation and holographic microscopy to measure rotational dynamics of non-spherical colloidal particles, J. Quant. Spectrosc. Radiat. Transfer 146, 499 (2014).

[39] M. A. Brown and E. J. Staples, Measurement of absolute particle-surface separation using total internal reflection microscopy and radiation pressure forces, Langmuir $\mathbf{6}$, 1260 (1990).

[40] J. R. Howse, R. A. L. Jones, A. J. Ryan, T. Gough, R. Vafabakhsh, and R. Golestanian, Self-Motile Colloidal Particles: From Directed Propulsion to Random Walk, Phys. Rev. Lett. 99, 048102 (2007).

[41] C. Bechinger, R. Di Leonardo, H. Löwen, C. Reichhardt, G. Volpe, and G. Volpe, Active particles in complex and crowded environments, Rev. Mod. Phys. 88, 045006 (2016).

[42] See Supplemental Material at http://link.aps.org/ supplemental/10.1103/PhysRevLett.125.238001 for experimental details on particle preparation and MSD analysis, as well as theoretical details on height-dependent diffusion coefficients, ionic diffusioosmosis along the wall, and activity-induced ypsotaxis.

[43] D. C. Henry, The cataphoresis of suspended particles. Part I. The equation of cataphoresis, Proc. R. Soc. A 133, 106 (1931).

[44] J. de Graaf, T. Peter, L. P. Fischer, and C. Holm, The raspberry model for hydrodynamic interactions revisited. II. The effect of confinement, J. Chem. Phys. 143, 084108 (2015).

[45] D. M. Huang, C. Sendner, D. Horinek, R. R. Netz, and L. Bocquet, Water Slippage Versus Contact Angle: A Quasiuniversal Relationship, Phys. Rev. Lett. 101, 226101 (2008).

[46] S. Eloul, W. C. K. Poon, O. Farago, and D. Frenkel, Reactive Momentum Transfer Contributes to the
Self-Propulsion of Janus Particles, Phys. Rev. Lett. 124, 188001 (2020).

[47] C. Zhou, H. P. Zhang, J. Tang, and W. Wang, Photochemically powered $\mathrm{AgCl}$ janus micromotors as a model system to understand ionic self-diffusiophoresis, Langmuir 34, 3289 (2018).

[48] D. B. Allan, T. Caswell, N. C. Keim, and C. M. van der Wel, trackpy: Trackpy v0.4.1, April 2018 https://doi.org/ 10.5281/zenodo.1226458.

[49] J. Palacci, C. Cottin-Bizonne, C. Ybert, and L. Bocquet, Sedimentation and Effective Temperature of Active Colloidal Suspensions, Phys. Rev. Lett. 105, 088304 (2010).

[50] H. Faxén, Einwirkung der Gefässwände auf den Widerstand gegen die Bewegung einer kleinen Kugel in einer zähen Flüssigkeit, Uppsala Universitet, 1921.

[51] C. W. Oseen, Neuere Methoden und Ergebnisse in der Hydrodynamik (Akademische Verlagsgesellschaft mb H., Leipzig, 1927).

[52] P. Sharma, S. Ghosh, and S. Bhattacharya, A high-precision study of hindered diffusion near a wall, Appl. Phys. Lett. 97, 104101 (2010).

[53] C. Ha, H. D. Ou-Yang, and H. K. Pak, Direct measurements of colloidal hydrodynamics near flat boundaries using oscillating optical tweezers, Physica (Amsterdam) 392A, 3497 (2013).

[54] A. J. Goldman, R. G. Cox, and H. Brenner, Slow viscous motion of a sphere parallel to a plane wall-I motion through a quiescent fluid, Chem. Eng. Sci. 22, 637 (1967).

[55] M.E. O'Neill, A slow motion of viscous liquid caused by a slowly moving solid sphere, Mathematika 11, 67 (1964).

[56] M. T. Kezirian, Hydrodynamics with a wall-slip boundary condition for a particle moving near a plane wall bounding a semi-infinite viscous fluid, Ph. D. thesis, Massachusetts Institute of Technology, 1992.

[57] C. van der Wel, R. K. Bhan, R. W. Verweij, H. C. Frijters, Z. Gong, A. D. Hollingsworth, S. Sacanna, and D. J. Kraft, Preparation of colloidal organosilica spheres through spontaneous emulsification, Langmuir 33, 8174 (2017).

[58] S. G. Flicker and S. G. Bike, Measuring double layer repulsion using total internal reflection microscopy, Langmuir 9, 257 (1993).

[59] A. Rashidi and C. L. Wirth, Motion of a janus particle very near a wall, J. Chem. Phys. 147, 224906 (2017).

[60] R. W. Verweij, S. Ketzetzi, J. de Graaf, and D. J. Kraft, Height distribution and orientation of colloidal dumbbells near a wall, arXiv:2009.14733.

[61] L. Coury, Conductance measurements part 1: Theory, Current Separations and Drug Development, Bioanalytical Systems 18, 91 (1999).

[62] C. van der Wel, N. Bossert, Q. J. Mank, M. G. T. Winter, D. Heinrich, and D. J. Kraft, Surfactant-free colloidal particles with specific binding affinity, Langmuir 33, 9803 (2017).

[63] S. J. Ebbens and J. R. Howse, Direct observation of the direction of motion for spherical catalytic swimmers, Langmuir 27, 12293 (2011).

[64] S. Ebbens, D. A. Gregory, G. Dunderdale, J. R. Howse, Y. Ibrahim, T. B. Liverpool, and R. Golestanian, Electrokinetic 
effects in catalytic platinum-insulator janus swimmers, Europhys. Lett. 106, 58003 (2014).

[65] Y. Ibrahim, R. Golestanian, and T. B. Liverpool, Multiple phoretic mechanisms in the self-propulsion of a Pt-insulator janus swimmer, J. Fluid Mech. 828, 318 (2017).
[66] A. T. Brown, W. C. K. Poon, C. Holm, and J. de Graaf, Ionic screening and dissociation are crucial for understanding chemical self-propulsion in polar solvents, Soft Matter 13, 1200 (2017).

[67] J. L. Anderson, Colloid transport by interfacial forces, Annu. Rev. Fluid Mech. 21, 61 (1989). 